\title{
Demand Sensitive Model for Tuning Price Over Satellite Digital Multimedia Broadcast System
}

\author{
Pasquale Pace, Associate Member, IEEE, and Zhili Sun, Member, IEEE
}

\begin{abstract}
In this paper an integrated satellite-terrestrial network architecture offering SDMB (Satellite Digital Multimedia Broadcast) services to mobile users through the satellite or terrestrial UMTS segment, is studied to test a novel Demand Sensitive Model (DSM).

The DSM is proposed for tuning price service over SDMB system to offer a price discount to large amount of users while maintaining profit for the service provider.

The simulations have been carried out to show the goodness of the proposed model within the system architecture; moreover the model, based on simple parameters, is very flexible and can be easily integrated in a "Price Adapter" module making the offered service attractive for end users and in the same time profitable for the satellite operator.
\end{abstract}

Index Terms-Demand sensitive model, direct broadcast satellites, dynamic pricing, UMTS, W-CDMA.

\section{INTRODUCTION}

$\mathbf{T}$ HE NUMBER of broadband users has been growing rapidly during the recent years. It is not only the number of users that increases but also the average data volume per user; as a consequence the demand for audio and video content is also increasing.

Broadcasting technology and services have been around almost as long as the radio communication itself, and have for more than half a century, been a "broadband" channel to the user. The development now is towards fully digital broadcast networks with return channels enabling out-reaching two-way communication in addition to traditional broadcast services.

Several initiatives and projects have emerged around the world to provide multimedia broadcast services using an hybrid satellite/terrestrial infrastructure to provide global coverage in a cost effective manner [1]-[3]. In the context of a growing market toward mobile multimedia, many studies on innovative concept of Satellite Digital Multimedia Broadcast (SDMB), have been conducted [4]-[6].

The purpose of the SDMB system is to provide capacity for several mobile operators to deliver cost effective streaming and download services directly to mobile handsets over satellite and terrestrial UMTS in both outdoor and indoor environments.

Manuscript received June 30, 2006; revised November 16, 2006. This work was supported by the European Commission within the frame of the IST/FP6 integrated project MAESTRO.

P. Pace is with the Department of Electronics, Computer and System Sciences (DEIS)—University of Calabria, Rende 87036, Italy (e-mail: ppace@ deis.unical.it).

Z. Sun is with the Centre for Communication Systems Research (CCSR) University of Surrey, Guildford GU2 7XH, U.K. (e-mail: z.sun@surrey.ac.uk).

Color versions of one or more of the figures in this paper are available online at http://ieeexplore.ieee.org.

Digital Object Identifier 10.1109/TBC.2006.889684
In addition, it aims at complementing $2 \mathrm{G}$ and $3 \mathrm{G}$ mobile systems by achieving true anywhere and anytime challenge with a dependable infrastructure offering point to point and point to multipoint services via satellite over zones not covered by the terrestrial networks.

Economy is another important issue when multicast or broadcast are introduced, both for the content provider and the network operator. Multicast and broadcast services have up to now been significantly more expensive than unicast services. The cost is related to network deployment, installation of customer premises equipment, management and maintenance. As the number of users increases it is expected that the cost per user will be less for broadcast or multicast than for unicast. For unicast each new receiver adds a constant cost, while for multicast the initial costs are higher but the cost of adding new receivers decreases proportional with increasing number of receivers. However the use of multicast only makes sense when the bandwidth savings is larger than the deployment and management cost, and for large groups multicast should be a profitable and useful service.

In a world that is so thoroughly changing because of the impact of communications services, the pricing of these services must play an important role so a lot of studies about pricing have been conducted in the last years.

A dynamic pricing mechanism was proposed in [7]. This adaptive pricing scheme assumes no knowledge of the demand function on the part of the network or the individual users. The scheme does not always converge, due to errors in users' expectations and errors in price estimates, but exponential smoothing of prices and demand estimates across periods ensures convergence for the $\mathrm{M} / \mathrm{M} / 1$ queue. Dynamic priority pricing has also been studied extensively by Gupta et al. [8], [9]. They have used an innovative approach based on dynamic programming to compare dynamic pricing with fixed prices. Given the computational intractability of this model, they used simulations to perform their assignments. Other authors have considered the pricing problem in the context of networks offering Quality of Service (QoS) guarantees. In [10] the authors show how the mobile users are more satisfied with the fact that they can choose whether they want to be served quickly by being priority users with high service charges or act as conventional users, who get the same normal rate with acceptable QoS degradation. In addition, the proposed pricing model is flexible enough to alleviate traffic congestion and meet expected revenue. In [11] the feasibility of dynamically adjusting prices based on congestion has been investigated; in particular, the authors ask whether congestion-based resource prices can be dynamically adjusted based on changes in demand and supply. They find that such dynamic pricing can be 


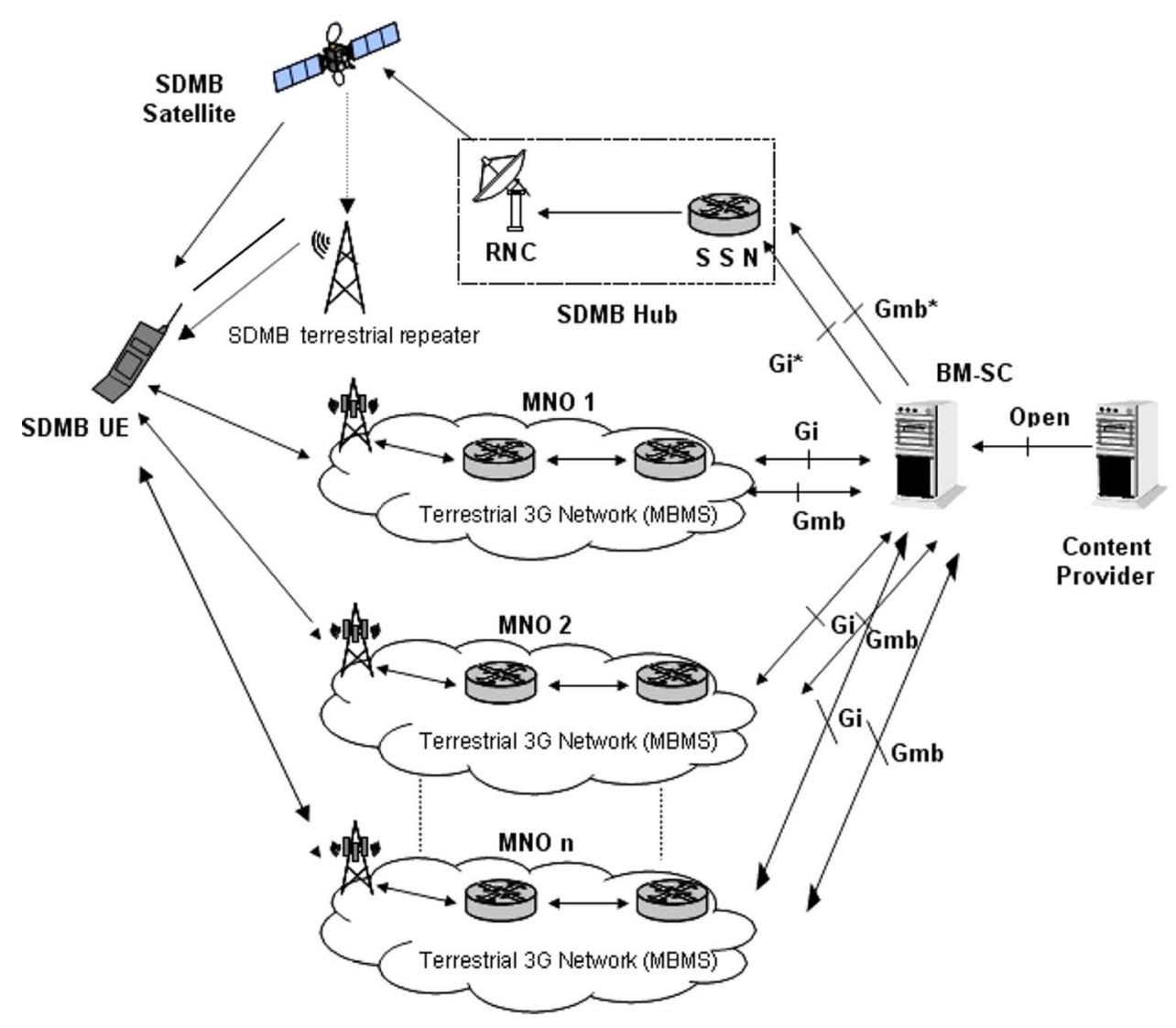

Fig. 1. Integrated system architecture.

accomplished using simple gradient algorithms, but that this approach requires a small amount of steady communication about demand and supply along each route.

We have already proposed and tested [12] a new efficient cost model for choosing the more suitable transmission bearer (satellite or terrestrial) in order to save service delivery cost using MAESTRO system architecture [1]; now we want to improve that model proposing a Demand Sensitive Model (DSM) for tuning price service over SDMB system trying to offer a price discount to the users when the amount of users asking for an SDMB service is large enough to guarantee a desired profit for the service provider.

Pricing can also be viewed as a mechanism by which the network operator communicates with own users and gives them incentives to use the network efficiently; this means that it can improve the value of services to users and provide stability and robustness.

The network framework architecture consists of bi-directional channel between the user equipment (UE) and the BM-SC (Broadcast Multicast-Service Center) which may be supported over either GPRS or UMTS-T bearer provided by the terrestrial Mobile Network Operator. Moreover, this architecture, also involves the ability to make use of multimedia broadcast/multicast services (MBMS) [13] provided by the terrestrial Mobile Network Operator in addition to the SDMB broadcast over satellite. In this way, a second option for routing of SDMB services to the end users is provided.

This paper first presents the system architecture, then explains the proposed Demand Sensitive Model showing the results ob- tained through simulation studies; finally concludes the paper and suggests future research directions.

\section{SYSTEM ARCHITECTURE OVERVIEW}

The system is based on a hybrid satellite \& terrestrial infrastructure operating in the IMT2000 core frequency band allocated to Mobile Satellite Systems. It makes use of the UTRA FDD WCDMA (UMTS Terrestrial Radio Air interface Frequency-Division Duplexing) waveform in full compliance with the relevant 3 GPP standard to allow a very low impact on the cost of $3 \mathrm{G}$ handset. The system relies on a very high power geo-stationary satellite able to overcome indoor penetration constraints in rural areas.

An high level overview of the generic SDMB architecture is shown in Fig. 1 developed in the EU F6 MAESTRO project [1]. It illustrates the key functional elements within the network that will be used to support the SDMB service. These elements include:

- SDMB-capable User Equipment; a dual mode T-UMTS/SUMTS mobile device enhanced to support SDMB services.

- SDMB Satellite that will support the transmission of SDMB services to defined coverage areas.

- SDMB Terrestrial Repeaters that may be deployed to enhance SDMB signal availability for UEs in urban areas by retransmitting the satellite signal on the ground.

- SDMB Hub that controls broadcast transmission over the SDMB satellite system taking as input media streams from the BM-SC. 
- BM-SC (Broadcast Multicast-Service Center) that controls all aspects of the delivery of SDMB services including authentication and authorization of subscribers, through delivery of services over the SDMB network, to service accounting.

- Content Provider that will provide the multimedia content to be delivered over the SDMB system.

Signaling between the terrestrial $3 \mathrm{G}$ network through the Gateway GPRS Support Node (GGSN) and BM-SC is exchanged using the Gmb interface. This represents the network side boundary of the MBMS Bearer Service from a control plane perspective. This includes user specific Gmb signaling and MBMS bearer service specific signaling in order to support inter-working and AAA (Authentication, Authorization and Accounting) functionalities. To allow the distribution of BM-SC functions, the Gmb protocol must support the use of proxies to correctly route the different signaling interactions in a manner which is transparent to the GGSN.

The Gi interface is used for sending and managing IP multicast ad broadcast datagram between the mobile network and the BM-SC providing several techniques for QoS control.

The $\mathrm{Gmb}^{*}$ and $\mathrm{Gi}^{*}$ interfaces towards the SDMB Hub are composed of a sub-set of the $\mathrm{Gmb}$ and $\mathrm{Gi}$ interfaces to support the functionality/attributes which are specific to the broadcast aspects of SDMB system, with some additional functionality to support a satellite node.

\section{LOCATION INFORMATION SIGNALING}

Location information must be sent to the BM-SC by each users in a new specific message after the reception of the service announcement broadcast message. In this way the BM-SC, could "periodically" estimate the number of connected users and their location implementing the following proposed strategy in order to offer a price discount to the users.

The following time message scheme shows the communication between the UE, the satellite Hub and the BM-SC highlighted the exchanged messages over different implicated interfaces:

1) Service Announcement message sent over the $\mathrm{Gi}^{*}$ interface between BM-SC and SDMB Hub

2) Service Announcement message forwarded through the SDMB to each UE by broadcasting

3) New Answer UE message sent to BM-SC via return channel over Terrestrial UMTS network in order to inform the BM-SC about the availability to receive the service. This message represents a new feature of the system architecture as fundamental parameter for the proposed DSM

4) Session start request message sent over $\mathrm{Gmb}^{*}$ interface

5) Session start response message sent over Gmb* interface

6) Data Transmission between BM-SC over Gi* interface

7) Data Transmission through the SDMB to each UE by broadcasting

How it is possible to observe in Fig. 2, message number 3 is a very fast message because it is sent through the terrestrial network whilst messages 2 and 7 are the only two messages that have a long delivery due to the satellite trip time.

Due to the long round trip time delay between satellite hub and user terminal, it is better to sent the session start response

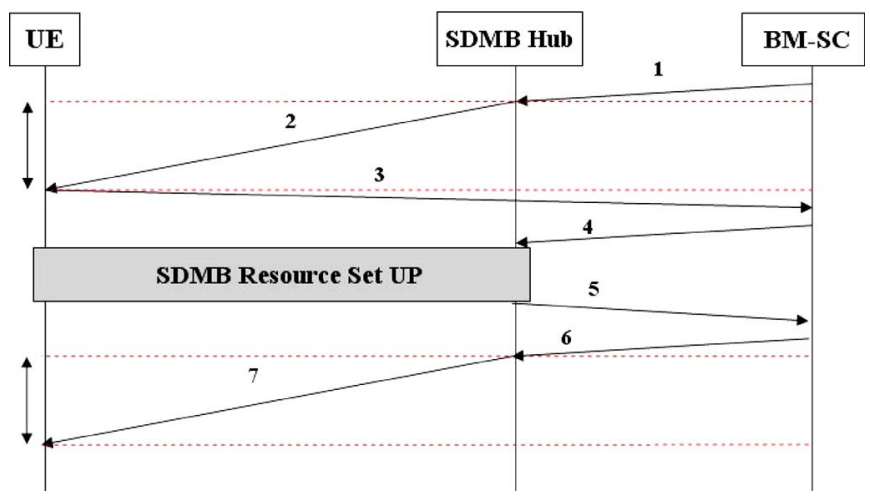

Fig. 2. Session start.

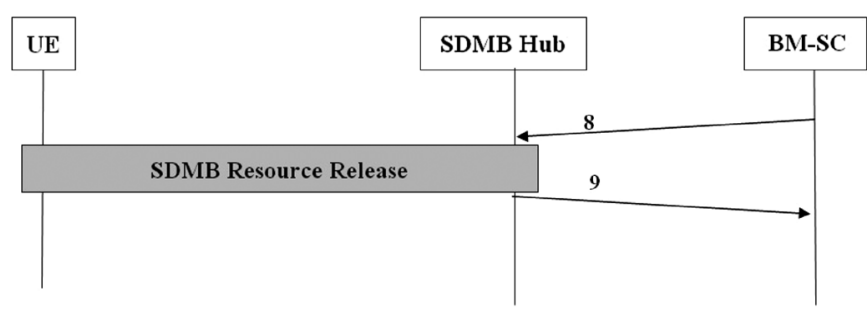

Fig. 3. Session stop.

message (5) after the SDMB Resource Set Up phase execution. In this way it is safely guaranteed that the necessary radio resources for the transfer of SDMB data to the interested UEs, will be established before the data transmission phase (6).

8) Session stop request message sent over $\mathrm{Gmb}^{*}$ interface

9) Session stop response message sent over $\mathrm{Gmb}^{*}$ interface (Fig. 3).

For the same reasons connected to the long round trip delay over the satellite link, this new time messages sequence must be adopted also in the session stop phase as shown in the previous figure.

\section{Demand Sensitive Model (DSM)}

Demand sensitive model is one of the pricing models that can be used for fast changes of prices in electronic commerce. Price setting algorithm for demand sensitive model helps service provider to get decision variables, price per unit that maximizes profit for the quantity ordered by clients [14].

In [12] we proposed a simple and efficient cost model for choosing the more suitable transmission bearer (satellite or terrestrial) in an integrated satellite-terrestrial UMTS architecture also considering the network congestion status. Now we want to adapt a demand sensitive model to our system architecture in order to offer a price discount to the users increasing the total profit for the network operator.

A number of pricing schemes have recently been proposed [15]. The Flat Pricing and the Proportional Fairness Pricing could seems suitable for our system architecture; nevertheless, they present some drawbacks as explained in the next sections.

\section{A. Flat Pricing}

Under a flat pricing scheme [16] the user is charged a fixed amount per time unit (e.g., month), irrespective of usage. This 
TABLE I

SDMB APPLICATIONS AND PARAMETERS

\begin{tabular}{|c|c|c|c|c|}
\hline $\begin{array}{c}\text { SDMB } \\
\text { Services }\end{array}$ & $\begin{array}{c}\text { Service } \\
\text { Example }\end{array}$ & Media & $\begin{array}{c}\text { MBMS User } \\
\text { Service } \\
\text { Classification }\end{array}$ & $\begin{array}{c}\text { Application } \\
\text { Data rate }\end{array}$ \\
\hline $\begin{array}{c}\text { Rich } \\
\text { media } \\
\text { Broadcast }\end{array}$ & $\begin{array}{c}\text { Audio } \\
\text { distribution } \\
\text { with low } \\
\text { quality video }\end{array}$ & $\begin{array}{l}\text { Stereo Audio, } \\
\text { Video (e.g. } \\
\text { 3fps) }\end{array}$ & Streaming & $\mathrm{Up}$ to $128 \mathrm{kbps}$ \\
\hline $\begin{array}{c}\text { Rich } \\
\text { media } \\
\text { Broadcast }\end{array}$ & $\begin{array}{c}\text { Video } \\
\text { distribution }\end{array}$ & $\begin{array}{c}\text { Video \& } \\
\text { supplementary } \\
\text { data (e.g. text, } \\
\text { still images) }\end{array}$ & Download & Up to $384 \mathrm{kbps}$ \\
\hline $\begin{array}{c}\text { Interactive } \\
\text { TV }\end{array}$ & $\begin{array}{c}\text { Video } \\
\text { streaming }\end{array}$ & $\begin{array}{c}\text { Video \& } \\
\text { supplementary } \\
\text { data (e.g. text, } \\
\text { still images) }\end{array}$ & Streaming & Up to $384 \mathrm{kbps}$ \\
\hline $\begin{array}{c}\text { Data } \\
\text { download }\end{array}$ & $\begin{array}{c}\text { General } \\
\text { Content } \\
\text { Distribution }\end{array}$ & $\begin{array}{l}\text { Video, Audio, } \\
\text { File Data } \\
\text { (binary data) }\end{array}$ & $\begin{array}{l}\text { Carousel, } \\
\text { download }\end{array}$ & Up to $384 \mathrm{kbps}$ \\
\hline $\begin{array}{c}\text { Data } \\
\text { download }\end{array}$ & $\begin{array}{c}\text { Audio } \\
\text { distribution } \\
\text { with low } \\
\text { quality video }\end{array}$ & $\begin{array}{l}\text { Stereo Audio, } \\
\text { Video (e.g. } \\
\text { 3fps) }\end{array}$ & Streaming & Up to $128 \mathrm{kbps}$ \\
\hline $\begin{array}{c}\text { Data } \\
\text { Download }\end{array}$ & $\begin{array}{c}\text { Audio } \\
\text { download }\end{array}$ & Stereo Audio & Download & Up to $48 \mathrm{kbps}$ \\
\hline $\begin{array}{l}\text { Group } \\
\text { Messages }\end{array}$ & $\begin{array}{l}\text { Reliable text } \\
\text { distribution } \\
\text { (eg. Local } \\
\text { news) }\end{array}$ & Text & Download & $\mathrm{Up}$ to $10 \mathrm{kbps}$ \\
\hline $\begin{array}{c}\text { Group } \\
\text { Messages }\end{array}$ & $\begin{array}{c}\text { Text } \\
\text { distribution } \\
\text { with images } \\
\text { and/or low } \\
\text { quality video }\end{array}$ & $\begin{array}{c}\text { Text, Still } \\
\text { images, Video } \\
\text { (e.g. 3fps) }\end{array}$ & $\begin{array}{l}\text { Carousel, } \\
\text { download }\end{array}$ & Up to $32 \mathrm{kbps}$ \\
\hline
\end{tabular}

pricing scheme has several desirable advantages. First and foremost, it is simple and convenient. Flat pricing makes no assumptions about the underlying network technology that is already deployed. Since charges are unrelated to usage, no measurements are required for billing and accounting. This also leads to social fairness in the sense that no distinction is made between poor and rich users. Provided that the flat price can be paid, anybody can access the network while receiving the same service level.

However, this scheme, even if it is easy to implement and very suitable for our telecommunication architecture, it does not provide any incentive or discount to the users that have no advantages to alter their transmission behavior to support the network' operation.

\section{B. Proportional Fairness Pricing}

This approach [17] consists in a whole system optimization that can be decomposed into a user and a network optimization, in this way a user chooses his/her price and the network allocates the rates according to the proportional fairness criterion. The resulting allocation of the rates, given the willingness to pay of each user, again solves the system's problem. The users optimally choose their prices and the network optimally assigns rates to the users. Although this scheme could be more attractive it is not suitable for our purposes because prices are fixed by the users according to the indicated willingness to pay for the service. The network keep track of the willingness to pay and allocate resources accordingly without offering any discount for the new users.

According to all the previous remarks, we propose a strategy of demand sensitive model based on the marginal decision rule; the marginal revenue must be equal to marginal cost when setting selling price. At this point selling price maximizes profit, production cost is at the lowest, and the unit price $P$ is the lowest when:

$$
P=f\left(E_{d}, M_{C}\right)
$$

where $E_{d}$ is the price elasticity of demand, and $M_{C}$ is the marginal cost. Marginal cost pricing is a particular form of usagebased pricing. The higher the demand for bandwidth, the lower the price the user is willing to pay for an additional unit of bandwidth. The demand function for bandwidth is thus downwards sloping.

This very general concept can be easily applied to our telecommunication system architecture assuming that there is one seller (the BM-SC) selling one or more types of SDMB services to the mobile users. The possible SDMB services are summarized in Table I. 
The seller wants to sell with highest profit, and lowest production cost. The seller would sell by the same price $P$ to all buyers willing to buy that type of commodity. The seller will set the first price per unit of service and as buyers increase, the price will be decreasing. The seller will accumulate the buyers' orders for service to sell in bulk at lowest price.

Let $T_{C}$ be the total production cost of a quantity $Q$, and $T_{R}$ the total revenue, then, seller's profit $(\pi)$ can be calculated as

$$
\pi=T_{R}-T_{C}
$$

where $T_{R}$ and $T_{C}$ are functions of $Q$.

A seller can get maximum profit when his production reaches the point where marginal revenue equals marginal cost, which is the first differential of profit with respect to quantity

$$
\frac{d \pi}{d Q}=\frac{d T_{R}}{d Q}-\frac{d T_{C}}{d Q}=0
$$

This marginal decision rule determines which price and amount of quantity demanded maximizes profit. The unit price of a service that yields maximum profit can expressed as a function of price elasticity of demand and marginal cost (see Appendix).

We would like to remark that the proposed price strategy is well suited to the proposed system architecture because the BM-SC can quickly collect all the UE's whishes to receive a specified service thanks to new answer message number 3 described in Section III.

\section{Application to the SDMB System}

In our scenario the $T_{C}$ value represents the total transmission cost using the satellite broadcast capability, it is not function of quantity $(Q)$ of the mobile users.

This can be evaluated using the following relation and it is related to the cost for the satellite channel usage per second $\left(C_{S}\right)$, the length $(L)$ of each multimedia SDMB service and the data rate $\left(R_{B S}\right)$ for broadcasting transmission [12]; therefore, the following equation represents the actual cost of the broadcast satellite transmission

$$
C_{\text {broad-SAT }}=C s \cdot \frac{L}{R_{B S}}=T_{C}
$$

After the evaluation of the satellite broadcast cost, it is possible to fix the price that each user would have to pay in order to obtain a good seller's profit.

Using relation (1) we know that the term TR (total revenue) is a function of the total number of users asking for an SDMB service and the price that each user pay for the service (see the Appendix). The BM-SC can evaluate the total number of users because receive a message from each UE during the session start phase as already explained; so the BM-SC is also able to evaluate the TR term in the equation choosing to reduce the service price if the number of asking users is higher than the number of users required for having the hoped seller's profit.

In a more complex architecture, a non linear Price Adaptor (PA) joined to an "utility function" can be supported by the

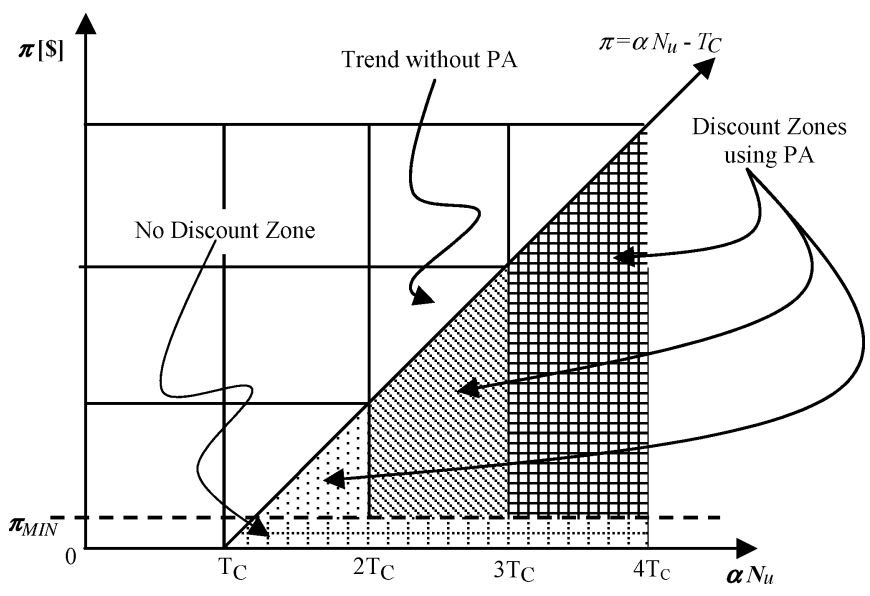

Fig. 4. Discount's zones using PA.

$\mathrm{BM}-\mathrm{SC}$ module in order to manage a dynamic pricing strategy depending on the amount of users asking for services.

The PA behavior can be based on the following consideration: the satellite operator can decide to reduce the price service for each users if the amount of users is enough for guaranteeing a good seller's profit.

the price reduction can be obtained by the proposed following equation:

$$
\text { PA choice } \begin{cases}\pi=\alpha N_{u}-T_{C} & \text { if } \alpha N_{u} \leq 2 T_{C} \\ \pi_{d}=\beta N_{u}-T_{C} & \text { if } \beta N_{u}>2 T_{C}\end{cases}
$$

where

$$
\beta=\left(1-\frac{\sqrt{N_{u}}}{W}\right) \alpha
$$

and $\alpha$ represents the reference selling price for each connection, $N_{u}$ is the number of users asking for a SDMB service and $\mathrm{W}$ is a weight parameter that will be changed during the simulation campaign in order to obtain a greater discount according to the amount of users.

The (4) also shows a fixed choice of the satellite seller that decided to decrease the service price if the total revenue is at least equal to double quantity of the satellite link cost $(2 T C)$; obviously we fixed this threshold for our studies but it depends on several business strategies and it can be modified inside the PA module.

In this last case, the total profit $\pi_{d}$ will be lower than the value obtained without the prices reduction, anyway, more users will be attracted by the price's discount thus the total profit for the satellite operator will be always greater than two time of the satellite link cost.

Fig. 4 shows a simple and general graphic representation of the PA working; $\pi_{M I N}$ represents the minimum threshold profit under which every service provider cannot apply any type of discount. The normal system behavior without the price adaptor is shown by the oblique line that also represents the maximum profit for the service provider; under this line there are several zones in which is possible to have a good profit applying the PA technique based on price's discount. 


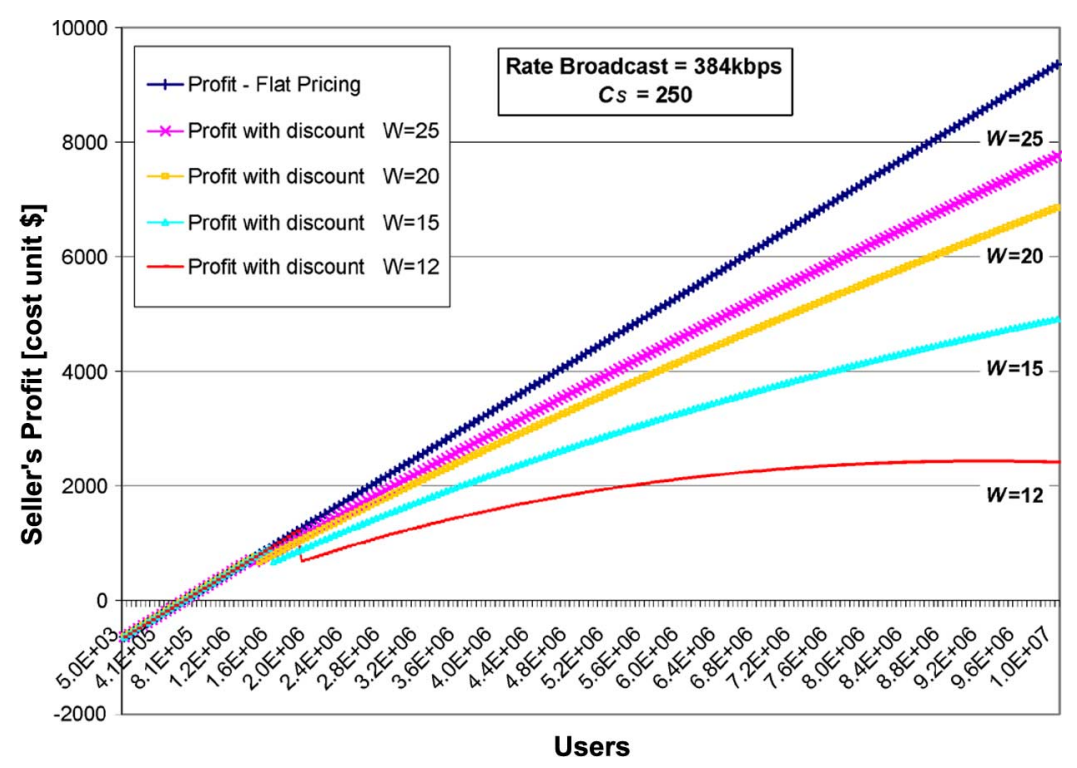

Fig. 5. Seller's profit vs. number of users.

According to these considerations, we propose in (5) a new mathematical relation for the estimation of the new discounted price; clearly the $\beta$ term is a pseudo-linear function of several parameters as the old price $\alpha$, the total number of users and the weight factor W. Moreover, from (4) and (5) it is directly possible to evaluate the $\beta$ term as a function of the total number of users, the weight factor $\mathrm{W}$ and the total production cost TC.

$$
\begin{aligned}
\frac{\beta N_{u}}{\left(1-\frac{\sqrt{N_{u}}}{W}\right)} & =2 T_{C} \Rightarrow \beta N_{u}=2 T_{C}-\frac{2 T_{C}}{W} \sqrt{N_{u}} \Rightarrow \beta \\
& =\frac{2 T_{C}}{N_{u}}\left(1-\frac{\sqrt{N_{u}}}{W}\right)
\end{aligned}
$$

\section{Simulation Results}

In our simulation campaign we perform a snapshot analysis (steady state) with maximum fixed number of MNOs (Mobile Network Operators) and users in each of them. In each snapshot one multimedia SDMB service is delivered to the recipients in the service area.

The service consists of delivery of a multimedia item $i$ that is characterized by its size in bits $L_{i}$ and number of recipients $N_{i}$. Each mobile user has a terminal that is always on and able to connect simultaneously to both terrestrial-UMTS (unicast-broadcast) and Satellite (broadcast) systems. We only consider the downlink segment. The main simulation parameters are summarized in Table II.

The maximum number of simultaneously active users for each MNO has been chosen according the following consideration; looking at a country like Italy, we know that the population is about 58 millions and there are 4 different mobile network operators; considering an homogeneous user's distribution between the operators with a user penetration rate of 0.85 we have about 12 millions of subscribers for each mobile network [18]. The user penetration rate is defined as the ratio of the number of mobile users to the total population of a certain geographical
TABLE II

SIMULATION PARAMETERS

\begin{tabular}{cc}
\hline \hline Cs & $250-500-750$ \\
\# of MNOs & 4 \\
Multimedia Service Size $L_{i}$ & 1 Mbits \\
Multimedia Service Data Rate & $384 \mathrm{kbps}$ \\
Maximum number of users for each MNO & 3 Millions \\
\hline \hline
\end{tabular}

area (e.g. a city area, a whole Country). Obviously, depending on different day's time, it can be possible to have a different number of users that are active at the same time but the worst case is about $25 \%$ of the total subscribers number. Supposing that each mobile network is well designed for managing own subscribers, the maximum number of active users has been chosen equal to 3 millions.

Introduction of multimedia services in mobile systems is important for mobile operators to maintain the Average Revепие Per User (ARPU) in the coming years, since voice service revenues are expected to decrease over the next decade. Putting the market into context, a recent report [19] forecasts 2006 spending levels for mobile content at around $\$ 3.3$ billion in Europe alone; according to this prevision, we present the results obtained using the value $384 \mathrm{kbps}$ for the $R_{B S}$ parameter without loss of generality.

Fig. 5 shows how is possible to evaluate the total amount of profit for the satellite operator varying the weight parameter $W$ and the number of users asking for the SDMB service. It is possible to observe that when the number of users is small, there is no profit for the satellite operator; this observation is perfectly agree with the use of satellite broadcast technology for providing services to a big number of users in a wide area.

When the number of users increases also the total seller's profit increases in a linear way according to the first part of (4) thus, when the number of users is big enough, the $P A$ module 


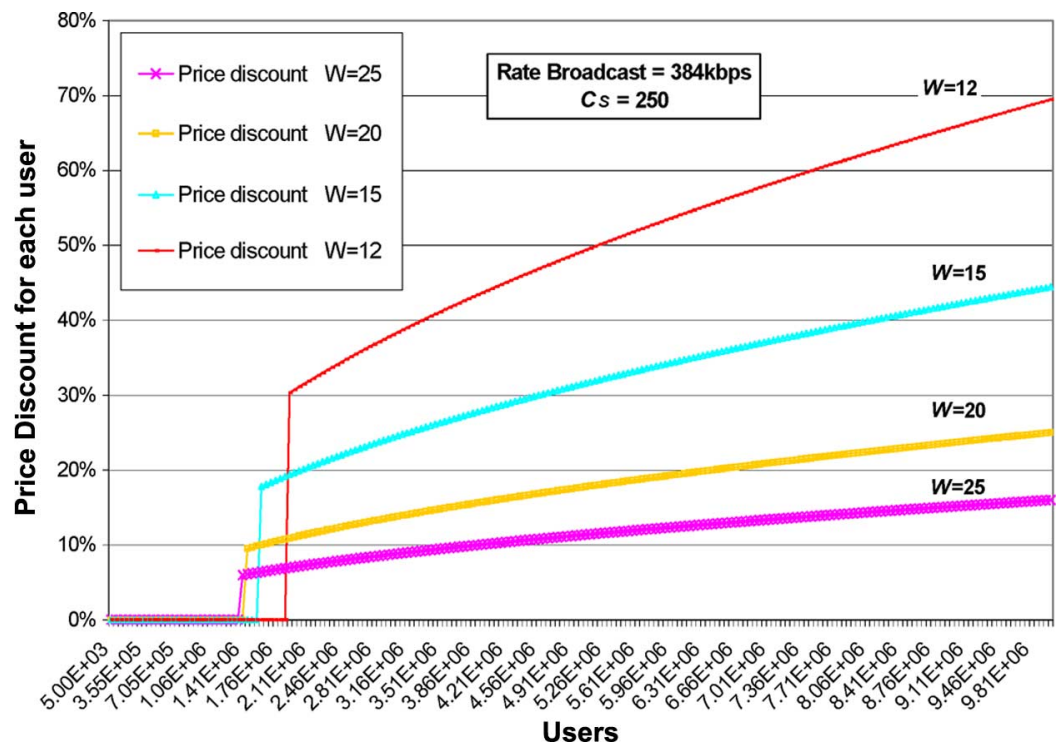

Fig. 6. Price discount varying the $W$ parameter $-C_{S}=250$.

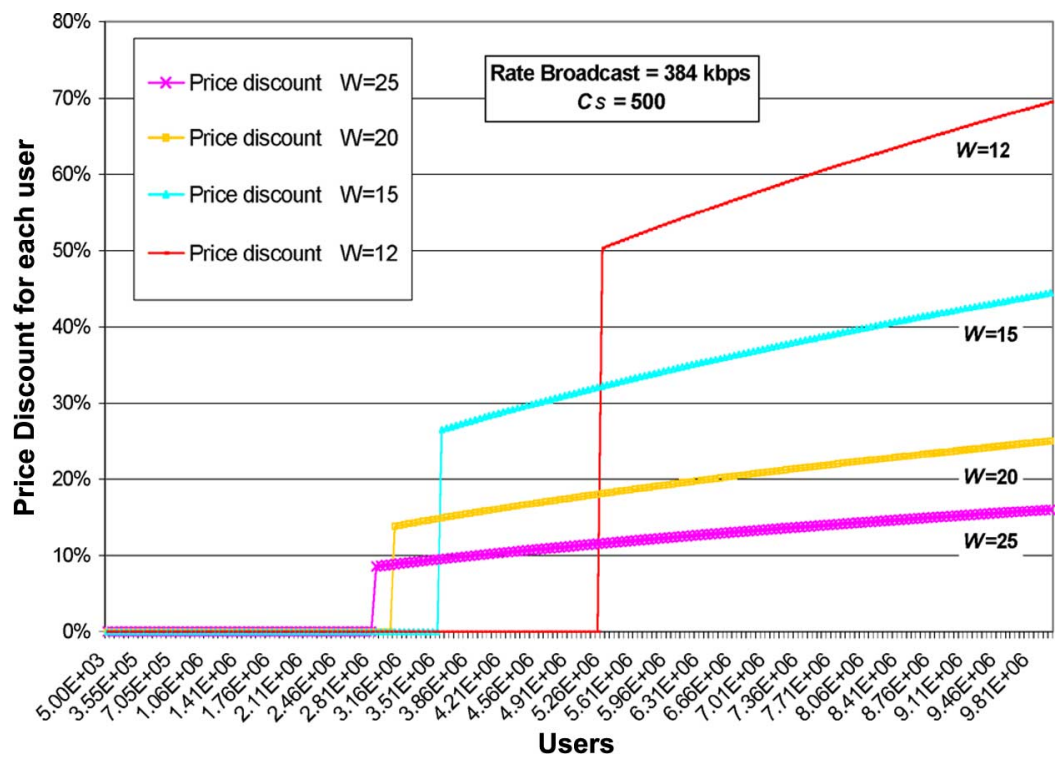

Fig. 7. Price discount varying the $W$ parameter $-C_{S}=500$.

tries to apply the prices discount according to the second part of the (4).

Great values of the $W$ parameter mean an high profit for the seller and a small discount for the single user as shown in Fig. 6. For this reason the proposed approach is very general because each satellite operator can choose a suitable value for the $\mathrm{W}$ parameter in its own price adapter module according to own need or business strategy, moreover, this parameter is very flexible and can be changed during the day or during some special periods in order to increase the total profit if the amount of users asking for a SDMB service grows very quickly.

Figs. 7 and 8 show how the price discount is also strictly related to the $C_{S}$ parameter that represents the broadcast transmission cost over the satellite link. It is possible to observe that when the value of this parameter increases, the SDMB system needs to have more users asking for an SDMB service in order to offer the same discount. Actually, referring to the Fig. 8, we can note that if the $C_{S}$ parameter is too big, no discount can be offered to the users when weight parameter $W$ is equal to 12 . This is not an uncommon behavior, it just means that even 10 millions of users are not enough to satisfy the discount condition in (4) guaranteeing that the total revenue for the satellite operator is at least equal to double quantity of the satellite link cost.

All the previous discussions demonstrate that the proposed model is very flexible and easily reconfigurable because it is based on few system parameters that can be appraised by the satellite operators.

In conclusion, the proposed price model is a very generic model adapted to the particular features of the MAESTRO system architecture in order to obtain good performances and attractive prices for the end mobile users; nevertheless, this model can be easily extended and personalized to the other telecommunication systems through a careful tuning of its simple parameters. 


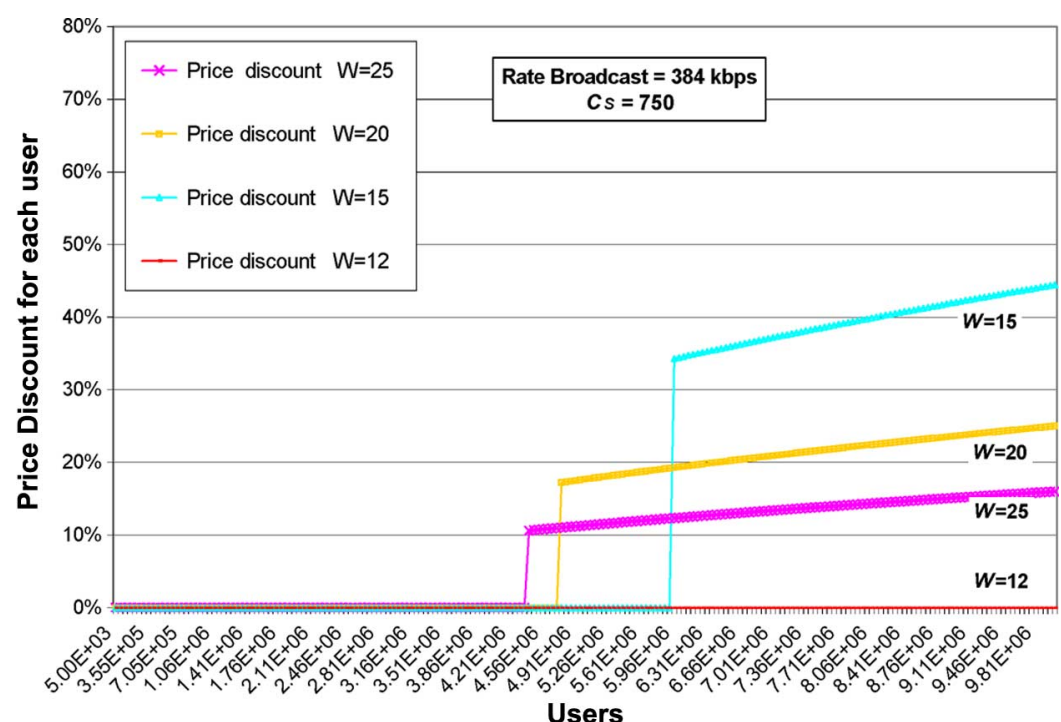

Fig. 8. Price discount varying the $W$ parameter- $C_{S}=750$.

\section{CONCLUSIONS}

This paper has proposed and tested a new effective Demand Sensitive Model (DSM) in order to offer a price discount to the users when the amount of users asking for an SDMB service is large enough to guarantee a desired profit for the satellite operator.

The conducted studies and the obtained results show how the new parameter $W$ is very flexible and can be easily used for tuning the price discount, making the offered service more attractive for the end users and more profitable for the satellite operator.

There are many technology aspects that must be taken into account in future researches, such as quality of service, multi-dimensional contracts, network mechanisms for conveying price information and the capabilities of edge devices to support new dynamic price models.

\section{APPENDIX \\ PRICE Elasticity OF DEMAND}

Price elasticity of demand is the most commonly used measure of consumers' sensitivity to price, pioneered by the great British economist Alfred Marshall [20], [21]. The price elasticity of demand, $E_{d}$ is defined as the magnitude of:

$$
\begin{aligned}
E_{d} & =\frac{\text { Percentage change in quality demanded }}{\text { Percentage change in price }} \\
E_{d} & =\frac{\Delta Q / Q}{\Delta P / P}=\frac{\Delta Q}{\Delta P} * \frac{P}{Q}
\end{aligned}
$$

where

$Q \quad$ Quantity demanded

$P \quad$ price per unit

$\Delta Q \quad$ change in quantity demanded

$\Delta P \quad$ change in price per unit
Price elasticity examines the relationship between price and unit sales, thereby assuming that the other factors that influence demand remain unchanged. Since the quantity demanded decreases when the price increases, this ratio is negative; however, the absolute value usually is taken and $E_{d}$ is reported as a positive number. Because the calculation uses proportionate changes, the result is a unit less number and does not depend on the units in which the price and quantity are expressed [22], [23] For demand-sensitive pricing model, price depends on the quantity demanded. In this case, quantity is independent while price is dependant.

From (2), marginal revenue $\left(M_{R}\right)$ can be expressed as

$$
\begin{aligned}
& M_{R}=\frac{d T_{R}}{d Q} \quad \text { where } T_{R}=P * Q \\
& M_{R}=\frac{d(P * Q)}{d Q}=P+Q \frac{d P}{d Q}=P\left(1+\frac{Q}{P} * \frac{d P}{d Q}\right)
\end{aligned}
$$

using the definition in (7), it is possible to write the marginal revenue in the following way

$$
M_{R}=P\left(1+\frac{1}{E_{d}}\right)
$$

Equating $M_{R}$ with marginal cost $\left(M_{C}\right)$ yields the profit maximizing relationship in terms of price and elasticity.

$$
M_{c}=P\left(1+\frac{1}{E_{d}}\right)
$$

Solving for selling price which maximizes profit yields

$$
P=\frac{E_{d}}{\left(E_{d}+1\right)} M_{c}
$$

The difference between price and marginal cost is a gross profit margin expressed as a percentage of prices. 


\section{ACKNOWLEDGMENT}

The authors would like to thank all MAESTRO project partners for their contributions to this work.

\section{REFERENCES}

[1] "Mobile Applications \& sErvices based on Satellite \& Terrestrial inteRwOrking project," [Online]. Available: http://ist-maestro.dyndns.org/MAESTRO/

[2] "Broadband Services for Everyone Over Fixed Wireless Access Networks: BROADWAN Project," [Online]. Available: http://www.telenor.no/broadwan/

[3] "Stratospheric Broadband CAPANINA Project," [Online]. Available: http://www.capanina.org/

[4] N. Chuberre, G. E. Corazza, M. G. Francon, C. Nussli, C. Selier, A. Vanelli-Coralli, and P. Vincent, "Satellite digital multimedia broadcasting for $3 \mathrm{G}$ and beyond $3 \mathrm{G}$ systems," in 13th IST Mobile \& Wireless Communications Summit 2004, Lyon, France, June 2004

[5] K. Narenhiran et al., "S-DMB system architecture and the MODIS DEMO," in IST Mobile and Wireless Telecommunications Summit, Aveiro, Portugal, June 2003.

[6] V. Kueh, M. Karaliopoulos, B. G. Evans, P. Vincent, G. Chengeleroyen, J. Malsch, N. Koutsokeras, E. Angelou, and I. Andrikopoulos, "Satellite Digital Multimedia Broadcast (SDMB) access layer definition," in 6th EMPS \& ASMS, Noordwijk/NL, September 2004, ESA/ESTEC.

[7] Y. Masuda and S. Whang, "Dynamic pricing for network service: equilibrium and stability," Management Science, vol. 45, no. 6, pp. 837-859, 1999.

[8] A. Gupta, D. O. Stahl, and A. Whinston, "A stochastic equilibrium model of internet pricing," Journal of Economic Dynamics and Control, vol. 21, pp. 699-702, 1997.

[9] — "The economics of network management," Communications of the ACM, vol. 42, no. 9, pp. 57-63, September 1999.

[10] S. Yaipairoj and F. C. Harmantzis, "Dynamic pricing with "alternatives" for mobile networks wireless," in Proceedings of IEEE Wireless Communications and Networking Conference, March 2004, vol. 2, WCNC, pp. 671-676.

[11] N. Jin, G. Venkitachalam, and S. Jordan, "Dynamic pricing of network resources," in Proceedings of IEEE Global Telecommunications Conference, GLOBECOM, December 2003, vol. 6, pp. 3216-3220.

[12] P. Pace, S. Marano, Z. Sun, and B. Evans, "Cost model for evaluation of SDMB service over integrated satellite-terrestrial UMTS networks," in Proceedings of 2nd International Symposium on Wireless Communication Systems-ISWCS, Siena, Italy, September 2005.

[13] 3GPP TS 22.246 V6.2.0 (2004-09) Technical SpecificationGroup Services and System Aspects, "Multimedia Broadcast/Multicast Service (MBMS) User Services," Stage 1 (Release 6).

[14] IEEE Computer Society Press,R. J. Kauffman and B. Wang, "New buyers' arrival under dynamic pricing market microstructure: the case of group-buying discounts on the Internet," in Proceedings of the 34th Hawaii Conference on Systems Science, Maui, Los Alamitos, CA, 2001.

[15] M. Falkner, M. Devetsikiotis, and I. Lambadaris, "An overview of pricing concepts for broadband IP networks," IEEE Communications Surveys, Second Quarter 2000.

[16] L. Anania and R. J. Solomon, "Flat: The Minimalist Price” Internet Economics, L. W. McKnight and J. P. Bailey, Eds. Cambridge, Massachusetts: MIT Press, 1997, pp. 91-118.

[17] F. Kelly, A. K. Maulloo, and D. K. H. Tan, "Rate control for communication networks: shadow prices, proportional fairness, and stability," Journal of the Operational Research Society, vol. 49, 1998.
[18] C. Campi, "Market analysis and demand forecast for mobile telephony services in Italy," in Proceedings of 15th ITS Biennial Conference Connecting Societies and Markets: Communication Technology, Policy and Impacts, Berlin, 2004.

[19] O. Beauvillain, "Paid content more successful on Mobile than on PC,' Jupiter MMXI, January 17, 2002.

[20] N. Dorward, The Pricing Decision: Economic Theory and Business Practice. London: Harper \& Row Publishers, 1987.

[21] J. D. Gwartney and R. L. Stroup, "Economics: private and public choice," in , 8th ed. Cambridge: Harvard University Press, 1997.

[22] B. P. Pashigian, "Price theory and applications: second edition," in . McCraw-Hill, 1998.

[23] B. M. Mitchell and I. Vogelsang, "Telecommunications pricing: theory \& practice," in . Cambridge: Cambridge University Press, 1992.

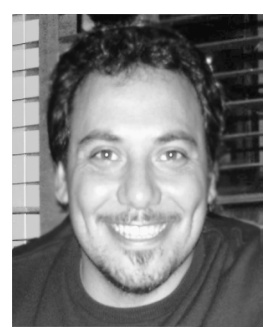

Pasquale Pace (A'05) received the M.S. degree in computer engineering and the Ph.D. degree in Information Engineering from University of Calabria, Italy, in 2000 and 2005, respectively.

From March 2005 to October 2005 he was visiting researcher at the Centre for Communication Systems Research (CCSR) at the University of Surrey-UK where he did research on multimedia satellite systems. Since November 2005 he joined the D.E.I.S Department, University of Calabria as Research Fellow.

Dr. Pace research interests include Multimedia Satellite Systems, DVB-RCSSatellite architectures, IP-Satellite, Mobility Management, Traffic \& Resource Management, Call Admission Control and integration of Satellite Systems and High Altitude Platforms in heterogeneous communications networks.

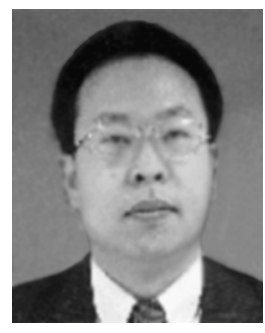

Zhili Sun (M'99) is Chair of Communication Networking in the Centre for Communication Systems Research (CCSR), Department of Electronic Engineering, School of Electronics and Physical Sciences, University of Surrey, UK. He received his BSc in Mathematics from Nanjing University, China and $\mathrm{PhD}$ in Computing Science from Lancaster University, UK. He was a Postdoctoral Researcher, from 1989 to 1993, in the Telecommunications Group, Queen Mary and Westfield College, University of London before coming to Surrey.

He has been a principal investigator and technical co-ordinator in many European projects including the ESPRIT BISANTE project on evaluation of broadband traffic over satellite using simulation approach, VIP-TEN project on Quality of Service (QoS) of IP telephony over satellite, EU 5th and 6th Framework Programme GEOCAST project on IP Multicast over satellites, ICEBERGS project on IP based Multimedia Conference over Satellite, SatLife project on IP over DVB-S/RCS, SATSIX project on IPv6 over satellite, Euro-NGI project on next generation Internet. He has also been a principal investigator in UK EPSRC, European Space Agency (ESA) and industrial projects on IP multicast security. He has supervised many PhDs and research fellows. He published a book titled "satellite networking" by Wiley and over 120 papers in International journals and conferences.

Professor Sun has also been a member of technical committee of international conferences and member of reviewers for EU and UK research proposal. $\mathrm{He}$ has acted as external examiner for $\mathrm{PhD}$ viva in many universities in $\mathrm{UK}, \mathrm{Eu}-$ rope, Singapore and China. He also teaches MSc, undergraduate and industrial courses on satellite networking, computer and data networks, Internet traffic engineering. 